\title{
Review Article \\ Human ABO Blood Groups and Their Associations with Different Diseases
}

\author{
Silamlak Birhanu Abegaz $\mathbb{B}$ \\ Woldia University, Faculty of Natural and Computational Sciences, Department of Biology, Ethiopia \\ Correspondence should be addressed to Silamlak Birhanu Abegaz; silamlakb@wldu.edu.et
}

Received 24 October 2020; Revised 18 January 2021; Accepted 20 January 2021; Published 25 January 2021

Academic Editor: Sercan Erg n

Copyright (c) 2021 Silamlak Birhanu Abegaz. This is an open access article distributed under the Creative Commons Attribution License, which permits unrestricted use, distribution, and reproduction in any medium, provided the original work is properly cited.

Introduction. Human $\mathrm{ABO}$ blood type antigens exhibit alternative phenotypes and genetically derived glycoconjugate structures that are located on the red cell surface which play an active role in the cells' physiology and pathology. Associations between the blood type and disease have been studied since the early 1900s when researchers determined that antibodies and antigens are inherited. However, due to lack of antigens of some blood groups, there have been some contentious issues with the association between the $\mathrm{ABO}$ blood group and vulnerability to certain infectious and noninfectious diseases. Objective. To review different literatures that show the association between ABO blood groups and different diseases. Method. Original, adequate, and recent articles on the same field were researched, and the researcher conducted a comprehensive review on this topic. Thus, taking out critical discussions, not only a descriptive summary of the topic but also contradictory ideas were fully retrieved and presented in a clear impression. In addition, some relevant scientific papers published in previous years were included. The article search was performed by matching the terms blood types/groups with a group of terms related to different diseases. The articles were screened and selected based on the title and abstract presented. Results. The susceptibility to various diseases, such as cancer, cardiovascular diseases, infections and hematologic disorders, cognitive disorders, circulatory diseases, metabolic diseases, and malaria, has been linked with ABO blood groups. Moreover, blood group AB individuals were found to be susceptible to an increased risk of cognitive impairment which was independent of geographic region, age, race, and gender. Disorders such as hypertension, obesity, dyslipidemia, cardiovascular disease (CVD), and diabetes were also more prevalent in individuals with cognitive impairment. Early etiological studies indicated that blood type $\mathrm{O}$ has a connection with increased incidence of cholera, plague, tuberculosis infections, and mumps, whereas blood type A is linked with increased incidence of smallpox and Pseudomonas aeruginosa infection; blood type B is also associated with increased incidence of gonorrhea, tuberculosis, and Streptococcus pneumoniae, E. coli, and salmonella infections; and blood type AB is associated with increased incidence of smallpox and E. coli and salmonella infections. Diabetes mellitus, hypercholesterolemia, arterial hypertension, and family history for ischemic heart disease are the most common risk factors for cardiovascular diseases and can be genetically transmitted to offspring. Higher incidence of cancers in the stomach, ovaries, salivary glands, cervix, uterus, and colon/rectum was common in blood type A people than in O type people. The link between the ABO blood type and thromboembolic diseases and bleeding risk are intervened by the glycosyltransferase activity and plasma levels and biologic activity of vWF (Von Willebrand factor), a carrier protein for coagulation factor VIII which is low in O type. Conclusion. Several studies related to the ABO phenotype show that genetically determined human $\mathrm{ABO}$ blood groups were correspondingly linked with an increased risk of various infectious and noninfectious diseases. However, further investigations are needed particularly on the molecular level of $\mathrm{ABO}$ blood groups and their association with various diseases.

\section{Introduction}

Human ABO blood type antigens exhibit alternative phenotypes and genetically derived glycoconjugate structures that are located on the red cell surface which play an active role in the cells' physiology and pathology $[1,2]$. Furthermore, oligosaccharide structures specific to the antigens defined the blood type. Thus, blood group antigens are secondary 
gene products, whereas various glycosyltransferase enzymes that help attach the sugar molecules to the oligosaccharide chain are primary gene products. These carbohydrate components are perceived as extraneous by the immune system of others and produce antibodies to them [3]. Medically, the $\mathrm{ABO}$ blood group system has been of great importance in different disease studies [4]. However, due to lack of antigens of some blood groups, there have been some contentious issues with the association between the $\mathrm{ABO}$ blood group and vulnerability to certain infectious and noninfectious diseases. The presence and absence of antigens in some blood types result in blood membrane alterations in both morphology and function. The functions dependent on the structure of blood types can associate the blood groups with diseases as well as health $[1,5]$. In addition to RBCs, blood group antigens can be found on leukocytes, certain tissues, plasma proteins, platelets, and various cell surface enzymes [6]. Blood group antigens can also be present in body fluids such as sweat, saliva, breast milk, seminal fluid, urine, gastric secretions, and amniotic fluid with soluble form [7, 8]. Most antigens however are the end product of a single gene, and modifications at the genetic level such as deletions, inversions, insertions, alternative splicing, or single-nucleotide polymorphisms (SNPs) lead to antigenic changes but can also give rise to new antigens or even complete loss of expression [3]. The ABO blood group antigens are possibly the most significant and intensively studied and were the first to be recognized [9]. The glycoconjugate structures on RBCs have many functions including receptors for exogenous ligands, viruses, bacteria, and parasites, transporters, channels, structural proteins, adhesion molecules, and enzymes [10]. The exact mechanisms that would explain the associations between blood group antigens and disease in adhesion molecules however have not yet known. But an unexpected number of these structures contribute to normal RBC development; some act as cell adhesion molecules (CAMs), and some play a role in human disease $[11,12]$. The ABO, Hh, Sese (secretor), and Lele (Lewis) genes play different roles in the final ABO antigen structure of an individual's body tissues as well as secretions [6, 7]. However, this basic evidence can be revised as new information was obtained [13]. The ABO blood group has been associated with many diseases and easily accessed in a patient's genetic makeup [14]. In the years 1960 and 1970, large epidemiological studies were carried out around the world and connections between the human $\mathrm{ABO}$ blood group and vulnerability to develop a number of diseases were broadly postulated [15]. ABO blood types were shown to have some associations with various infectious [7, 16] and noninfectious diseases [7, 17]. Environmental and host genetic factors may be imperative in the genesis of diseases. During 1901 Landsteiner's discovery, the ABO blood group system was studied as an etiological factor of many diseases, for example, carcinoma of the stomach and peptic ulcer [18]. ABO antigens are thought to be evolutionarily beneficial in conferring resistance against pathogens. However, the susceptibility to various diseases, such as cancer, cardiovascular diseases, infections, and hematologic disorders, has been linked with ABO blood groups [19]. Mostly $A$ and $B$ antigens are secreted by the cells and are found in human blood circulation. Therefore, nonsecretors are at risk to a variety of infections [20]. The likely pathogenesis for this vulnerability is that many organisms may bind to polysaccharides on the cell surface but soluble blood group antigens may block this binding $[16,21]$. On the one hand, [22] reported that the $\mathrm{ABO}$ alternative phenotype can prevent the species carrying it from being threatened by a pathogen using a given carbohydrate as a receptor. On the other hand, the $\mathrm{ABO}$ polymorphism leads to a polymorphic production of anti-A and anti-B natural antibodies, which potentially protect individuals from several infectious agents expressing $A$ and $B$ motifs [23]. In view of the above possible associations of the $\mathrm{ABO}$ blood group and different diseases, the objective of this paper is thus to review different literatures that show the association between $\mathrm{ABO}$ blood groups and different diseases.

\section{Methodology}

Original, adequate, and recent articles on the same field were researched, and the researcher conducted a comprehensive review on this topic. Thus, taking out critical discussions, not only a descriptive summary of the topic but also contradictory ideas were fully retrieved and presented in a clear impression. In addition, some relevant scientific papers published in previous years were included. The article search was performed by matching the terms blood types/groups with a group of terms related to different diseases. The articles were screened and selected based on the title and abstract presented.

\section{Association of ABO Blood Groups and Diseases}

3.1. Infectious Diseases. According to the study of [24-26], $\mathrm{ABO}$ polymorphism has been associated with certain infectious diseases. The presence/absence of $\mathrm{A} / \mathrm{B}$ antigens and correspondent absence/presence of anti-A/B antibodies provide strong/weak defensive lines against infection. Many vertebrate species uphold the $\mathrm{ABO}$ gene and hence benefited from it. However, having both functional $\mathrm{A}$ and $\mathrm{B}$ genes in common within species may not be important because they may lose anti-A/B antibodies in the long run. But frequent gene conversion of $\mathrm{A} / \mathrm{B}$ specificity producing amino acid substitutions or recombination with nonfunctional partial genes may have conferred an adaptation against microbial attacks [27]. Glycoconjugate red cell surfaces are used effectively by parasites, bacteria, and viruses as receptors for attachment [9]. Because infectious agents often use cell surface glycoconjugates as receptors for attachment, glycosylation polymorphisms in the $\mathrm{ABO}$ blood type may affect host-pathogen connections and result in vulnerability difference among individuals with diverse glycosylation profiles [9]. It should be recalled that certain microbial parasites share blood group antigens with their hosts (molecular mimicry). An association between $\mathrm{ABO}$ and infectious diseases like cholera was identified by early etiological studies [9]. According to the study of [28], the major variations observed in $\mathrm{ABO}$ blood groups in different parts of the world were due 
to the epidemics that have occurred in the past. The presence of an "H-like" antigen on the plague bacillus (and cholera) and an "A-like" antigen on the smallpox virus was suggested as some major differences. This would make individuals who can make anti-H (A1 and B) more resistant to the plague and cholera and individuals who have anti-A (groups B and O) more resistant to smallpox $[1,29]$. Reports showed that once a person is infected with cholera (Vibrio cholerae strains $\mathrm{O} 1$ $\mathrm{El}$ Tor and O139), the O blood types had a greater frequency of severe infections than the non-O blood types [26]. Patients of blood type $\mathrm{O}$ were more vulnerable to infections like gastrointestinal outbreaks caused by Escherichia coli $\mathrm{O} 157$ in Scotland in 1996, and a total of $87.5 \%$ of blood type O patients died. According to the study of [30], blood type $\mathrm{O}$ was also associated with increased incidence of cholera, plague, tuberculosis infections, and mumps, whereas blood type A was associated with increased incidence of smallpox and Pseudomonas aeruginosa infection; blood type B is also associated with increased incidence of gonorrhea, tuberculosis, and Streptococcus pneumoniae, E. coli, and salmonella infections; and blood type $\mathrm{AB}$ is associated with increased incidence of smallpox and E. coli and salmonella infections. The Norovirus, a strain-dependent pathogen, however, is likely to have less risk of infection and symptomatic disease in blood group B individuals, but blood group $\mathrm{O}$ individuals have a much greater risk of infection $[1,9]$. The expression of Le and $\mathrm{ABH}$ antigens in the gastrointestinal tract is also closely linked to vulnerability of Norovirus infection. Nonsecretors appear more susceptible to infections by Haemophilus influenzae, Neisseria meningitides, [7, 30], and Streptococcus pneumoniae and urinary tract infection caused by E. coli [31]. Peptic ulcer also has a connection with the ABO blood group, and it was the first to be identified. Blood type $\mathrm{O}$ individuals showed that they had higher susceptibility to peptic ulcers $[1,9]$. Gastritis and ulceration of the stomach/duodenum were later correlated with infection with the bacterium Helicobacter pylori. It was also found that antibiotics and acid secretion inhibitors cured patients from peptic ulcer by eradicating the bacteria causing it [30, 32]. It was reported that $H$. pylori attachment to the human gastric mucosa was mediated by the $\mathrm{H}$ type 1 and Lewis $\mathrm{b}\left(\mathrm{Le}^{\mathrm{b}}\right.$ ) fucosylated antigens. The soluble glycoproteins presenting Le $\mathrm{b}^{\mathrm{b}}$ or antibodies to the Le $\mathrm{L}^{\mathrm{b}}$ antigen could inhibit the bacterial binding. Furthermore, the conversion of $\mathrm{Le}^{\mathrm{b}}$ to $\mathrm{ALe}^{\mathrm{b}}$ by the addition of a terminal GalNAc weakened the bacterial binding. This may explain the decreased infectivity of blood type $\mathrm{AB} / \mathrm{B} / \mathrm{A}$ individuals as compared to blood type $\mathrm{O}$ individuals $[7,9]$. Infections related to bacterial meningitis is another example of infectious disease which can be linked to the ABO phenotype. Three species of bacteria, N. meningitides, $H$. influenza, and $S$. pneumoniae, cause about $75 \%$ of all bacterial meningitis; the capsules of these bacteria contain polysaccharide antigens, which the immune defense of the host must recognize and respond to with the appropriate antibodies [7, 33].

3.2. Cognitive Disorders. Higher levels of plasma coagulant glycoproteins, vWF (Von Willebrand factor), and FVIII (factor VIII) in non-O blood group individuals have been connected with greater risk of dementia and cognitive impairment, suggesting that coagulation factors may play a role in these disorders $[7,34]$. Blood group $\mathrm{AB}$ individuals were found to be susceptible to an increased risk of cognitive impairment $(\mathrm{OR}=1.82)$ as revealed by a large prospective case-control study, which was independent of geographic region, age, race, and gender [7]. In this study, the FVIII levels differed significantly among blood groups with the order $\mathrm{AB}>\mathrm{B}>\mathrm{A}>\mathrm{O}[7,35]$, and disorders such as hypertension, obesity, dyslipidemia, cardiovascular disease (CVD), and diabetes were also more prevalent in individuals with cognitive impairment, which points out that a common etiology is likely; the blood group influences CVD risk, and CVD risk factors are known to be connected with dementia and cognitive impairment. Surprisingly, blood types $\mathrm{AB}$ and $\mathrm{B}$ were more frequent in Blacks (in both cases and controls) [7, 35], which may be an overlooked factor in increased rates of stroke and CVD in this population.

3.3. Circulatory Diseases. Factually, several studies reported that non-O blood groups are linked with the high risk of ischemic heart disease and emergence of atherosclerosis indicators [36]. Moreover, peripheral vascular disease, venous thromboembolism, myocardial and angina infarction, and these connections were checked by subsequent GWAS (genome-wide association studies) and further confirmed in 2008 with a systematic review and meta-analysis [1, 37]. Genetic factors also contribute to the development of coronary artery disease (CAD) and influence individual response to risk factor modification. The most significant cardiovascular risk factors, diabetes mellitus, hypercholesterolemia, arterial hypertension, and family history for ischemic heart disease, are, at least in part, genetically transmitted. Of these, family history very accurately predicts coronary events [1, 38]. ABO blood types are genetically transferred as well, through chromosomes 9 at locus 9q34. The ATP-binding cassette 2 (ABCA2) genes were located at locus 9q34 which plays a role in cholesterol homeostasis. Furthermore, genetic variation considerably affects the risk for developing CAD which has been discovered recently on the 9p21 chromosomal section. Much of the hereditary aggregation of CAD can be linked to genetic risk factors. Therefore, inheritance of ABO blood types could play a significant role in this context $[9,38]$. ABO blood type antigens are connected to the protein backbone of coagulation factors vWF and FVIII and hence undoubtedly affect coagulation. To be sure, blood type $\mathrm{O}$ patients are prone to excessive blood loss because of just about 25\% lower concentrations of vWF and FVIII coagulation factors in the plasma, which is the result of the increased avoidance of these glycoproteins; the phenomenon is related to the $\mathrm{H}$ antigen linked to their backbone [26]. On the other hand, the higher plasma concentrations of coagulation factors vWF and FVIII in non-O blood type persons have been connected with the greater risk for ischemic heart disease and thromboembolic disease $[1,26]$. Blood type $A B$ patients with preeclampsia have an increased risk of severe, early-onset, or intrauterine growth restriction (IUGR) forms. Preeclampsia, the leading cause of maternal and fetal 
morbidity and mortality during pregnancy, was unique and likely to be associated with maternal blood type. Placental protein 13 (PP13) is a galectin (galectin-13) that binds to beta-galactosides like $\mathrm{N}$-acetyl-galactosamine, galactose, and fucose, located at end positions on ABO blood type antigens. PP13 is also reflected as an early marker for preeclampsia. PP13 is mainly produced by the placenta in anthropoid primates and is predominantly localized to the syncytiotrophoblast apical membrane, where it can be secreted and/or shed into the maternal circulation [19]. Similarly, ABO was linked to myocardial infarction [9].

3.4. Cancer. Even though the association between the $\mathrm{ABO}$ blood type and cancer was the subject of rigorous investigation in the mid-1900s, there has been improved attention after the contemporary publication of reports establishing a connotation between the $\mathrm{ABO}$ blood type and pancreatic cancer [39-41]. Blood type antigens play a role in tumorigenesis, metastasis, and prognosis and are probably take part in cell recognition, cell signaling, and cell adhesion [42]. Expression of $\mathrm{ABH}$ and related antigens differs during cellular development, aging, and differentiation; this is mainly realistic during carcinogenesis and pathological phenomena [9]. ABH antigens can be found in the epithelial tissues of the gastrointestinal tract, breast, uterine cervix, mouth, lung, bladder, and prostate. But these antigens are missing from the glycolipids and glycoproteins of malignant tissues in these areas [31]. For example, it is thought that DNA methylation in the promoter region for the blood group A gene may inhibit transcription of the associated enzyme and therefore loss of the A antigen, but different mechanisms for the reduction of mRNA have been found in A tumors, which appear to be specific to each tumor cell line [43]. Loss of A and $\mathrm{B}$ antigens leads to metastasis, resulting from downregulated transcription of $\mathrm{ABO}$ with related loss of $\mathrm{A}$ or $\mathrm{B}$ transferase activity, and upsurges the buildup of other antigens which act as ligands for selectins and facilitate the metastatic process [31]. As normal antigens are lost, malignancy develops and tumor antigens are acquired; the decrease in $\mathrm{A}, \mathrm{B}$, and $\mathrm{H}$ antigens is indirectly proportional to the metastatic potential of the tumor [30]. Procoagulant and angiogenic properties of blood type antigens are known, act as ligands for selectins, and increase cellular motility and resistance to apoptosis; these biological roles may assist tumor development, and a model has been proposed that may account for the described relations between the presence or absence of these markers and the outcome of disease [44]. Some non-A blood type people have tumors with real A antigens or with "A-like" antigens that have very similar properties to A antigens; in these people, the tumor antigens would be seen as foreign and would interact with anti-A antibodies, resulting in attack of the tumor $[7,30]$. This explains why a greater incidence of cancer is common in blood type A people than in blood type O people; the A or "A-like" properties of these tumor antigens are not recognized as foreign in blood type A people [30]. Blood type A people have a greater occurrence of cancer in the stomach $(22 \%)$, ovaries $(28 \%)$, salivary glands (64\%), cervix (13\%), uterus (15\%), and colon/rectum (11\%) as compared to blood type O people
$[7,30]$. It is important to note that although $\mathrm{ABO}$ genotypes are considerably associated with the risk of certain cancers, they do not cause cancer; they only indicate susceptibility [9]. In the reverse, lack of association does not confer protection: many studies have been unsuccessful to find the link between the blood type and breast cancer $[7,45,46]$.

3.4.1. Leukemia and Lymphoma. A and B antigens were usually decreased till they are untraceable in patients with acute leukemia and aplastic anemia; as the patient's condition improves, the antigens increase again to their former levels [30]. However, the loss of antigens may be due to an inhibitory factor related to antigen-antibody binding or an abnormal distribution or density of antigen sites in the RBC membrane but not deficiency in transferase synthesis or activity [7, 47]. Expression of A, B, or $\mathrm{H}$ antigens in leukemia patients had significantly lowered between $17 \%$ and $37 \%$ when compared to healthy controls of A, B, or AB. Patients with myeloid malignancies had reduced expression of $\mathrm{A}$ or $\mathrm{B}$ antigens. Blood type $\mathrm{O}$ patients had reduced $\mathrm{H}$ antigens by $55 \%$ and $21 \%$ when compared with healthy controls of the same ABO genotype [31]. Primary central nervous system lymphoma (PCNSL) begins in and characteristically remains limited to the central nervous system (CNS) in non-Hodgkin's lymphoma [7, 48], while secondary central nervous system lymphoma (SCNSL) characteristically does not originate from the CNS but may later take part in the CNS up to $10 \%$ to $30 \%$ of cases $[7,49]$. A study of 36 patients with PCNSL occurrence reported $8.3 \%$ in blood type A, $27.8 \%$ in blood type $\mathrm{B}, 55.6 \%$ in blood type $\mathrm{O}$, and $8.3 \%$ in blood type AB [48] whereas another study that assessed 202 patients with secondary central nervous system lymphoma (SCNSL) indicated that the occurrence was $5.0 \%$ in blood type A, $61.9 \%$ in blood type B, 29.7\% in blood type O, and $3.5 \%$ in blood type $\mathrm{AB}[7,49]$. The same populations of healthy controls were also used for both studies; the shared blood type percentages were $22.2 \%$ in blood type B, 37.1\% in blood type $\mathrm{A}, 6.1 \%$ in blood type $\mathrm{AB}$, and $35.6 \%$ in blood type $\mathrm{O}[48,49]$. There are very few studies of the association between $\mathrm{ABO}$ blood types and children with leukemia and lymphoma. A study of pediatric victims with acute myeloid leukemia (AML; $n=116$ ), acute lymphoblastic leukemia (ALL; $n=522)$, Hodgkin's lymphoma $(n=63)$, and nonHodgkin's lymphoma $(n=78)$ exhibited an important variation in the overall dissemination of blood groups when compared to the source population for all but the AML victims [7, 48]. This study stated that the occurrence of Hodgkin's lymphoma was $45.6 \%$ greater in blood type B victims and $56.5 \%$ lesser in blood type A patients; the occurrence of nonHodgkin's lymphoma was $52.9 \%$ lesser in blood type A patients; the occurrence of ALL was $14.3 \%$ greater in blood type $\mathrm{O}$ victims; but there was no variation in the dissemination of blood types in victims with AML [7, 49]. A distinct multicenter pediatric study of 682 victims with ALL and 224 victims with AML stated that the occurrence of ALL was $56.5 \%$ greater in blood type $\mathrm{O}$ victims, $35.8 \%$ lesser in blood type A victims, and $26.9 \%$ lower in blood type B victims, while the occurrence of AML was $28.8 \%$ greater in blood type A victims [7]. 
3.4.2. Stomach Cancer. It is the fourth most public cancer worldwide and the second leading cause of cancer deaths; reliable studies since the 1950s have shown that blood type A persons have about a $20 \%$ higher risk of stomach cancer than blood type $\mathrm{O}$ individuals [50]. The 2012 metadata analysis showed that type A persons had an odds ratio of 1.11 and type $\mathrm{O}$ persons had an odds ratio of 0.91 for gastric cancer; in addition, blood type A persons had significantly greater rates of $H$. pylori infection than non-A blood type victims (odds ratio $=1.42$ ) $[7,51]$. This is important because a current study of $\mathrm{ABO}$ blood types and $H$. pylori found that the risk of advanced precancerous gastric lesions was considerably affected by the presence or absence in the bacterial DNA of two SNPs in the cytotoxin-associated gene $A(\operatorname{Cag} A)$,

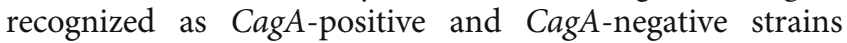
$[7,52]$. Thus, $A B O$ antigens on the gastric epithelium are binding sites for the $H$. pylori bacterium, which then injects $\operatorname{CagA}$ virulence protein into the cellular cytoplasm $[7,52]$.

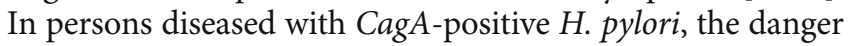
was considerably greater in blood type $\mathrm{A}$ than in blood group $O$ for intestinal metaplasia $(O R=1.36)$ and dysplasia $(\mathrm{OR}=1.78)$, with a collective $\mathrm{OR}$ of 1.42 , whereas in those with CagA-negative strains or who were not diseased with H. pylori, blood type A persons had a considerably lesser danger than blood type $\mathrm{O}$ persons for intestinal metaplasia and dysplasia $(\mathrm{OR}=0.60)[7,52]$.

3.4.3. Pancreatic Cancer. It is the seventh most common cause of cancer death worldwide and is one of the most destructive cancers, with death rates nearly equal to occurrence rates [50]. Blood type $\mathrm{A}, \mathrm{B}$, and $\mathrm{AB}$ persons have a $25 \%$ higher risk of gastric and pancreatic cancer, as well as $17 \%$ higher risk of pancreatic cancer only; as compared to blood type $\mathrm{O}$, the vulnerability of exocrine pancreatic cancer is uppermost in blood type B (odds ratio, 1.72) and lower for blood types $\mathrm{AB}$ (odds ratio, 1.51) and $\mathrm{A}$ (odds ratio, 1.32) [7, 9]. Secretor status had insignificant consequence on this risk, but the behavior of $H$. pylori, which is also influenced by the blood type, may have an effect on risk [7, 31]. Non-O blood type persons diseased with CagA-negative $H$. pylori have greater risk for pancreatic cancer $(\mathrm{OR}=2.78)$ [53]. Non-O blood type persons diseased with CagA-negative H. pylori have greater risk for pancreatic cancer (odds ratio $=2.78$ ) [50]. The greater rate of pancreatic cancer in non-O blood type persons also has been explained by a genome-wide association study [53] which found that the SNP rs505922 mapped to the first intron of the ABO blood type gene in the $9 q 34$ locus and was in complete linkage disequilibrium with the O/non-O allele [7, 45]. Another study also found that blood group $\mathrm{O}$ is intensely linked with pancreatic disease in patients with von Hippel-Lindau (VHL) syndrome and is considerably associated with solid pancreatic lesions in victims with pancreatic neuroendocrine tumors (PNETs); VHL victims have a great risk of evolving multiple tumors throughout the body, and their risk of developing both benign and malignant PNETs is $8 \%$ to $17 \%[7,42]$.

3.4.4. Multiple Endocrine Neoplasia Type I (MENS-1). It was detected in 105 patients, among which 46 (43.8\%) were iden- tified with a neuroendocrine tumor; from these 46 sufferers, 14 had several forms of tumor for the entire 60 tumors; it was found in the lung $(n=5)$, stomach $(n=3)$, gallbladder $(n=1)$, duodenum $(n=13)$, pancreas $(n=34)$, or thymus $(n=4)$ [7, 42]. Of the 17 patients with metastatic tumors, 16 of them (93.8\%) had blood type O, whereas of the 43 benign tumor patients, 32 of them $(74.4 \%)$ were blood type $\mathrm{O}$; of the 46 neuroendocrine tumor patients, 35 of them (76.1\%) had blood type O, 9 of them (19.6\%) were blood type A, 2 of them (4.3\%) were blood type B, and none were blood type $\mathrm{AB}$. Of the 59 nonneuroendocrine tumor patients, only 31 of them (52.5\%) were blood type O, 15 of them $(25.4 \%)$ were blood type A, 7 of them (11.9\%) were blood type B, and 6 of them $(10.2 \%)$ were blood type $\mathrm{AB}[7,42]$. Blood type $\mathrm{AB}$ is found in $4 \%$ of the overall population and was found in $3.8 \%$ of the study cohort, so the absence of type $A B$ in patients with neuroendocrine tumors was notable $[7,42]$.

3.4.5. Cancer of the Rectum/Colon. Augmented activity of the $\alpha 1,4$-fucosyltransferase of the FUT3 gene (Lewis) and $\alpha 1,2$ fucosyltransferase of the FUT2 gene (secretor) seems to take part in the development and regulation of cancers of the distal colon; type 2 as well as type 1 chains and Lewis antigens are generally existing in the fetal colon and vanished in healthy adults, but they reemerge in grown persons who have distal colon cancers [7, 31, 54]. Only type 1 chains are expressed by secretors in the normal colon; however, type 1 or type 2 chains are not common by nonsecretors in normal or cancerous colon tissue [55]. Secretor status also determines whether the $\mathrm{H}$ antigen (blood group $\mathrm{O}$ ) can be seen in normal and cancerous colon tissue $[7,55]$. The goblet cell which is the secretory part of the normal colon is crucial for the expression of blood type antigens [54]. Blood type A antigen is rarely seen on malignant tumors of type $\mathrm{O}$ or type $\mathrm{B}$ persons; about $10 \%$ of colon tumors of homozygous type $\mathrm{O}$ people express A antigen and have $\mathrm{N}$-acetylgalactosaminyltransferase activity $[7,31]$.

3.5. Metabolic Diseases. Since metabolic diseases are multifactorial and are not controlled by a single gene or antigen, the role of blood types in metabolic disease is most likely to be complex. However, some fascinating relationships have been found and presented below.

3.5.1. Hypertension. It can have several causes; thus, it is not surprising that different studies have found various links between blood type antigens and hypertension. Some investigations indicate that the rate of hypertension in blood type $\mathrm{B}$ was maximum, followed by blood type $\mathrm{A}$ and blood type $\mathrm{AB}$ which had the lowest rate of hypertension [56]. Another study also reported that there was a link between blood type A and systolic blood pressure in Caucasians but not in Blacks $[7,57]$. In hypertension due to abnormal red blood cells' sodium and potassium transport, no connection was found with the Rh, ABO, Kidd, Duffy, MNS, or P blood types or with the major histocompatibility HLA antigens $[7,58]$. In hypertension due to abnormal red cells' lithium-sodium counter transport, no connection was found with the MNS blood type polymorphism [7, 59]. When secondary forms 
of hypertension can be ruled out, essential hypertension is possibly diagnosed. However, it can also be diagnosed due to atherosclerotic or fibromuscular etiology, renal stenosis (renovascular hypertension), or primary aldosteronism linked with little plasma renin levels; people with these circumstances were compared to normotensive controls and persons with secondary hypertension, and insignificant changes were obtained in ABO, Rh, Kidd, Kell, Duffy, P, haptoglobin, PGM-1, or acid phosphatase systems [56, 58]. Conversely, there were important alterations in the incidences of the MNS blood type antigens when comparing normotensive controls with persons who had essential or renovascular hypertension; when compared to normotensives, essential hypertensives were considerably dissimilar among Whites, whereas the same alteration was not seen among Blacks [58]. Three clearly different phenotypic occurrences were seen when persons with atherosclerotic renovascular hypertension were compared to essential hypertensives and normotensives $[56,58]$.

3.5.2. Hyperlipidemia. Investigators have also studied the relationship between $\mathrm{ABO}$ blood type antigens and hyperlipidemia. Some study stated that LDL cholesterol, total cholesterol, and triglycerides were higher while HDL cholesterol was lower, in blood types $A$ and $B$; however, blood type $A B$ was protective for hyperlipidemia [56]. Other studies also informed that blood type A was linked with LDL cholesterol and higher total cholesterol, but there is no relation with HDL cholesterol [7, 57]. The most exciting discovery was the relationship of $\mathrm{ABO}$ and secretor blood types with serum levels of intestinal alkaline phosphatase (I-ALP) and apo lipoprotein B-48 (apo B-48); I-ALP is necessary for the passage of chylomicrons from bowels to the circulation and is thus an indicator for chylomicron absorption, whereas apo B-48 is a protein that strengthens the chylomicron membrane and is thus an indicator for chylomicron fabrication $[7,60]$. There are important variations in serum I-ALP and apo B-48 between blood type $\mathrm{O}$ and $\mathrm{B}$ secretors and all other blood types; the $\mathrm{O}$ and $\mathrm{B}$ secretors have very high serum levels of these indicators relative to blood type $A / A B$ secretors and nonsecretors of all blood types $[7,60]$. ABO nonsecretors alone have about $20 \%$ of the serum I-ALP of secretors, and among secretors, blood type A has very low activity $(2.8 \pm 1.1 \mathrm{IU} / \mathrm{L}$; mean $\pm \mathrm{SEM})$ compared to blood types $\mathrm{B}$ and $\mathrm{O}(14.1 \pm 1.1 \mathrm{IU} / \mathrm{L}$ and $19.0 \pm 2.5 \mathrm{IU} / \mathrm{L}$, respectively) [7]. It is believed that I-ALP is linked with ABO antigens on RBCs of nonsecretors and is also accumulated by the A antigens of secretors, thus being quickly removed from circulation in these persons, while the soluble circulating antigens of $\mathrm{O}$ and $\mathrm{B}$ secretors favorably link with I-ALP and protect its removal in these persons [60]. Blood type A persons also have lesser serum apo B-48 levels, which can be due to a genetic downregulation of I-ALP activity in their intestines, subsequently lower chylomicron secretion [7, 60], and possibly lesser serum cholesterol levels.

3.5.3. Diabetes Mellitus. A huge prospective study conducted in France indicates that there was no connection between the Rh blood type and risk of type 2 diabetes mellitus (T2DM).
However, individuals with blood type $\mathrm{O}$ shows the lowermost risk of T2DM, whereas those with blood type $\mathrm{B}$ were at the uppermost risk, followed by type $\mathrm{AB}$ and type $\mathrm{A}$ individuals; nevertheless, the risk for type $\mathrm{AB}$ people did not have statistical implication $[7,61]$. When $\mathrm{Rh}$ and $\mathrm{ABO}$ types were assessed together, blood type $\mathrm{B}^{+}$persons showed the uppermost risk, followed by type $\mathrm{AB}^{+}, \mathrm{A}^{-}$, and $\mathrm{A}^{+}$persons, but similar risk was seen for the other types $[7,61]$. When adjusted for metabolic covariates (fasting blood glucose and lipids), blood type $\mathrm{AB}$ persons showed the maximum risk of T2DM (odds ratio, 1.95), followed by type B (odds ratio, 1.26) and type A (odds ratio, 1.21) as compared with blood type O persons, who had the lowest risk [61]. Other findings also indicated inconsistent results: a study in Yemen indicated that the maximum arbitrary blood sugar and insulin levels were found in blood type $A$, whereas blood type $A B$ showed a defensive effect [56]. A research in Iraqi persons indicated greater blood glucose, total cholesterol, and blood pressure in blood type $\mathrm{O}$ persons, followed by lesser risk in type $\mathrm{A}, \mathrm{B}$, and $\mathrm{AB}$ persons, who showed the bottommost risk $[7,61]$. A large study in Bangladesh indicated that there was no relationship between $\mathrm{ABO}$ blood types and T2DM. A study in Malaysia also shows lesser risk of T2DM in blood types $\mathrm{A}$ and $\mathrm{O}[62]$. It has also been reported that nonsecretors are more likely to have T2DM $[7,57]$. Undoubted confirmation has described a genetic relationship in those of European ancestry between nonsecretor status (se/se; homozygous for the A/A alleles of the FUT2 gene) and insulindependent type 1 diabetes mellitus (T1DM) [7]. The odds ratio for nonsecretor status was $1.29\left(p=7.3 \times 10^{-14}\right)$ in the case of the control population, while the relative risk for nonsecretor status was $1.22\left(p=6.8 \times 10^{-6}\right)$ in the diabetic family population and the combined results clearly indicated a locus for T1DM in the FUT2 gene $\left(p=4.3 \times 10^{-18}\right)$ [7]. The I-ALP findings by blood type for diabetes were the same as the findings for hyperlipidemia, with considerably greater serum IALP and entire ALP levels in blood type B and O secretors (together with controls) when compared to A secretors or $\mathrm{ABO}$ nonsecretors, but no substantial variation in serum IALP or entire ALP between A secretors and ABO nonsecretors (together with controls) [7, 33]. However, when comparing blood type $\mathrm{B}$ and $\mathrm{O}$ secretor diabetics to controls, I-ALP activity was similar between type 1 and type 2 diabetics but considerably greater in both types than in the controls; likewise, there was insignificant variation in I-ALP activity between type 1 and type 2 diabetics in the A secretor and $\mathrm{ABO}$ nonsecretor groups, but both types of diabetics had greater I-ALP activity than the controls in these groups [7, 33]. Further comparisons were made between the diabetics and controls in the group $\mathrm{B}$ and $\mathrm{O}$ secretors: mostly, fasting I-ALP activity was greater in the diabetics than in the controls; both types of diabetics had considerably greater liver ALP activity than the controls; type 2 diabetics had greater liver ALP than type 1 diabetics; and type 2 diabetics also had additional abnormal ALT and GGT values than type 1 diabetics $[7,33]$. Troubled liver function could weaken the clearance of I-ALP by the liver and would elucidate the greater ALP levels found in the diabetics; high I-ALP has been described in victims with cirrhosis of the liver [7]. 
3.6. Malaria. The connection between the ABO blood type and malaria was first suggested by $[1,63]$; they indicate that type $\mathrm{B}$ provides a selective advantage to malarial disease. By 1978, a noticeable number of type A patients were known from combined data analysis as compared to types B and O. However, malaria types were rarely stated in the literature [9]. According to the ABO phenotype, there are some variations in Lewis antigen level among A, B, and AB blood types, since these blood types carry fewer Le antigens than $\mathrm{O}$ types, because their corresponding transferases use similar precursors $[1,64]$. The latest assumption has stated that P. falciparum malaria can be the reason for the three-fold greater incidence of the Le (a_b_) phenotype among people of African ancestry [25]. However, ABO blood types' role to defend against malaria has not been given much consideration; it has been revealed that blood type $\mathrm{O}$ can provide confrontation to severe $P$. falciparum malaria by reducing rosetting mechanisms $[1,65]$. Severe malarial pathogenesis was linked to rosetting and sequestration. The first implies the formation of masses of diseased RBCs and normal RBCs and/or platelets. The second implies the course whereby $P$. falciparuminfected RBCs move on and stick to the microvascular endothelium and then are removed from the circulation [9]. The microcirculatory blockade by cytoadhesion can decrease oxygen and substrate supplies [9]. The complement receptor type 1 (CR1) is a rosetting receptor in RBCs, and CR1 seems to carry the Knops blood type antigens with the S1 (a) phenotype creating less rosettes [66]. Identification of a carbohydrate structure found in glycophorin A or B that consists of sialic acid and galactose is the major requisite for the entrance of Plasmodium falciparum merozoites into human RBCs [1, 67]. Sialic acid is common to some pathways that use glycophorins as ligands have also been related to $\mathrm{ABO}$ phenotypes. Sialic acid is one of the vital molecules for parasite attachment, and it has been thought that the humanchimpanzee variations in malaria vulnerability are linked to the genetic alterations in sialic acid molecules found in glycophorins [68]. In areas extremely endemic for Plasmodium falciparum malaria, it is well known that a variety of $\mathrm{RBC}$ polymorphisms related to confrontation to severe illness have experienced positive selection [69]. Overall, P. vivax accounts for $40 \%$ of cases [70]. The Duffy blood type system, which has 6 discrete antigens, is involved in P. vivax infections; mutations of the FY gene that result in RBCs without the Duffy antigen protect against infection by this strain of malaria [70].

\section{Conclusions}

Even though many studies have proven the association between $\mathrm{ABO}$ blood types and diseases by describing possible mechanisms, others did not confirm it and making the exact decision falls into uncertainty due to inconsistent results. Nevertheless, evidences were collected here to make this supposition clear. ABO may influence the risk of different diseases by different known and unknown mechanisms. It is now clear that $\mathrm{ABO}$ blood types are not the exact cause of diseases, but they can be susceptible and surrender to disease and health problems. In general, non-O blood types are more susceptible to diseases than O. It can be useful to increase the knowledge of persons in this aspect because individuals with high risk blood types could be screened and trained for modifying their lifestyles, health behavior, and environment and other attempts that may increase public health. The importance of human blood types can be seen more clearly in the context of population movement and the persistent combat between humans and infectious disease. Evidence for selection by infectious diseases at the level of the ABO and secretor genes is persuasive, but for other blood group antigens, founder effects appear more likely to account for the distribution of blood group polymorphisms except for parts of the world in which malaria is endemic. Available data suggests that survivals from malaria have been the most significant selective force acting on the blood groups. Moreover, further investigations have to be made particularly on the molecular level of ABO blood groups and their association with various diseases.

\section{Conflicts of Interest}

The author declares that he has no conflicts of interest.

\section{Acknowledgments}

The author acknowledged the researchers who had investigated human $\mathrm{ABO}$ blood groups and associated diseases as well as health benefits to combat cancer-related diseases, cardiovascular disease, circulatory diseases, infectious disease, malaria, metabolic diseases, and so on.

\section{References}

[1] Abdulganiyu, "Distribution of ABO and Rh (D) blood groups and associated traits: a study of the College of Nursing and Midwifery," Msc Thesis Dissertation, Kogi State, Obangede, 2016.

[2] N. Mohandas and A. Narla, "Blood group antigens in health and disease," Current Opinion in Hematology, vol. 12, no. 2, pp. 135-140, 2005.

[3] G. A. Denomme, "Molecular basis of blood group expression," Transfusion and Apheresis Science, vol. 44, no. 1, pp. 53-63, 2011.

[4] T. Chandra and A. Gupta, "Association and distribution of hypertension, obesity and $\mathrm{ABO}$ blood groups in blood donors Iranian," Journal of Paediatric Haematology Oncology, vol. 2, no. $4,2012$.

[5] S. G. Sandler and D. Mallory, "Biological functions of blood groups in health and disease," Haematologia (Budap)., vol. 27 , no. 1, pp. 1-13, 1995.

[6] C. Green, "The ABO, Lewis and related blood group antigens; a review of structure and biosynthesis," FEMS Microbiology Immunology, vol. 1, no. 6-7, pp. 321-330, 1989.

[7] R. Ewald and S. Sumner, "Blood type biochemistry and human disease," Wiley Interdisciplinary Reviews. Systems Biology and Medicine, vol. 8, no. 6, pp. 517-535, 2016.

[8] G. Daniels, "Human Blood Groups," in Blackwell Science, Oxford, 2nd edition, 2002. 
[9] F. Yamamoto, E. Cid, M. Yamamoto, and A. Blancher, "ABO Research in the Modern Era of Genomics," Transfusion Medicine Reviews, vol. 26, no. 2, pp. 103-118, 2012.

[10] J. P. Cartron and Y. Colin, "Structural and functional diversity of blood group antigens," Transfusion Clinique et Biologique, vol. 8, no. 3, pp. 163-199, 2001.

[11] M. J. Telen, "Erythrocyte Adhesion Receptors: Blood Group Antigens and Related Molecules," Transfusion Medicine Reviews, vol. 19, no. 1, pp. 32-44, 2005.

[12] F. A. Spring and S. F. Parsons, "Erythroid cell adhesion molecules," Transfusion Medicine Reviews, vol. 14, no. 4, pp. 351363,2000 .

[13] W. T. Morgan and W. M. Watkins, "Unravelling the biochemical basis of blood group ABO and Lewis antigenic specificity," Glycoconjugate journal, vol. 17, no. 7-9, pp. 501-530, 2000.

[14] K. Akhtar, G. Mehdi, R. Sherwani, and L. Sofi, "Relationship Between Various Cancers And ABO Blood Groups - A Northern India Experience," The Internet Journal of Pathology, vol. 13, no. 1, 2010

[15] H. Mäkivuokko, S. J. Lahtinen, P. Wacklin et al., “Association between the $\mathrm{ABO}$ blood group and the human intestinal microbiota composition," BMC Microbiology, vol. 12, p. 94, 2012.

[16] S. D. Jefferys and C. A. Kenneth, "Transfusion biology and therapy," in Principles and Practice of Infectious Diseases, G. L. Mandell, Ed., p. 708, Churchill Livingston, Philadelphia, 6th edition, 2005.

[17] T. Umit, E. N. Tiftik, U. Sakir, G. Ozrur, I. K. Tamer, and C. Handan, "Relationship between $\mathrm{ABO}$ blood group and skin," Dermatology Online Journal, vol. 11, no. 3, pp. 1-6, 2008.

[18] A. B. Lutfullah, N. U. S. Quraishi, A. Hanif, B. Z. Khan, and I. M. Bukhshi, "Association of ABO blood groups and major ischaemic heart disease risk factors," Annals of King Edward Medical University, vol. 16, no. 3, pp. 189-193, 2010.

[19] N. G. Than, R. Romero, H. Meiri et al., "PP13, Maternal ABO Blood Groups and the Risk Assessment of Pregnancy Complications," PLoS ONE, vol. 6, no. 7, p. e21564, 2011.

[20] A. E. Naeini, M. Rostami, and S. E. Naeini, "Chronic viral hepatitis and their relation to ABO blood groups and rhesus (Rh) factor," Medical Case Studies, vol. 1, no. 1, pp. 5-7, 2010.

[21] P. G. C. Odeigah, "Influence of blood group and secretor genes on susceptibility to duodenal ulcer," East African Medical Journal, vol. 67, no. 7, pp. 487-500, 1990.

[22] G. Daniels, "Blood group polymorphisms: molecular approach and biological significance," Transfusion Clinical Biology, vol. 4, no. 4, pp. 383-390, 1997.

[23] F. Calafell, F. Roubinet, A. Ramírez-Soriano, N. Saitou, J. Bertranpetit, and A. Blancher, "Evolutionary dynamics of the human ABO gene," ABO gene Human Genetics, vol. 124, no. 2, pp. 123-135, 2008.

[24] T. Boren, P. Falk, K. A. Roth, G. Larson, and S. Normark, "Attachment of Helicobacter pylori to human gastric epithelium mediated by blood group antigens," Science, vol. 262, no. 5141, pp. 1892-1895, 1993.

[25] C. M. Cserti and W. H. Dzik, "The ABO blood group system and Plasmodium falciparum malaria," Blood, vol. 110, no. 7, pp. 2250-2258, 2007.

[26] D. J. Anstee, "The relationship between blood groups and disease," Blood, vol. 115, no. 23, pp. 4635-4643, 2010.
[27] F. Yamamoto, E. Cid, M. Yamamoto, N. Saitou, J. Bertranpetit, and A. Blancher, "An integrative evolution theory of histoblood group _ABO_ and related genes," Scientific Reports, vol. 4, no. 1, 2015.

[28] A. E. Mourant, A. D. A. Kopec, and K. Domanieswska-Sobezek, The Distribution of the Human Blood Groups and Other Polymorphisms, Oxford University Press, London, 2nd edition, 1976.

[29] G. Garratty, "Relationship of Blood Groups to Disease: Do Blood Group Antigens Have a Biological Role?," in American Red Cross Blood Services Southern, California, 2005.

[30] G. Garratty, "Blood groups and disease: a historical perspective," Transfusion Medicine Reviews, vol. 14, no. 4, pp. 291301,2000

[31] G. Daniels, "Functional aspects of red cell antigens," Blood Reviews, vol. 13, no. 1, pp. 14-35, 1999.

[32] W. Dickey, J. S. Collins, R. G. Watson, J. M. Sloan, and K. G. Porter, "Secretor status and Helicobacter pylori infection are independent risk factors for gastroduodenal disease," Gut, vol. 34, no. 3, pp. 351-353, 1993.

[33] C. C. Blackwell, "The role of ABO blood groups and secretor status in host defences," FEMS Microbiology Immunology, vol. 1, no. 6-7, pp. 341-349, 1989.

[34] K. S. Alexander, N. A. Zakai, S. Gillett et al., “ABO blood type, factor VIII, and incident cognitive impairment in the REGARDS cohort," Neurology, vol. 83, no. 14, pp. 1271-1276, 2014.

[35] N. A. Zakai, S. E. Judd, K. Alexander et al., “ABO blood type and stroke risk: the REasons for Geographic And Racial Differences in Stroke Study," Journal of Thrombosis and Haemostasis, vol. 12, no. 4, pp. 564-570, 2014.

[36] A. Amirzadegan, M. Salarifar, S. Sadeghian, G. Davoodi, C. Darabian, and H. Goodarzynejad, "Correlation between $\mathrm{ABO}$ blood groups, major risk factors, and coronary artery disease," International Journal of Cardiology, vol. 110, no. 2, pp. 256-258, 2006.

[37] G. L. Daniels, A. Fletcher, G. Garratty et al., "Blood group terminology 2004: from the International Society of Blood Transfusion committee on terminology for red cell surface antigens," Vox Sanguinis, vol. 87, no. 4, pp. 304-316, 2004.

[38] C. Carpeggiani, M. Coceani, P. Landi, C. Michelassi, and A. L. Abbate, "ABO blood group alleles: A risk factor for coronary artery disease. An angiographic study," Atherosclerosis, vol. 211, no. 2, pp. 461-466, 2010.

[39] B. M. Wolpin, A. T. Chan, P. Hartge et al., "ABO Blood Group and the Risk of Pancreatic Cancer," JNCI Journal of the National Cancer Institute, vol. 101, no. 6, pp. 424-431, 2009.

[40] S. Iodice, P. Maisonneuve, E. Botteri, M. T. Sandri, and A. B. Lowenfels, "ABO blood group and cancer," European Journal of Cancer, vol. 46, no. 18, pp. 3345-3350, 2010.

[41] B. M. Wolpin, P. Kraft, M. Gross et al., "Pancreatic Cancer Risk and ABO Blood Group Alleles: Results from the Pancreatic Cancer Cohort Consortium," Cancer Research, vol. 70, no. 3, pp. 1015-1123, 2010.

[42] A. B. Weisbrod, N. Nilubol, L. S. Weinstein et al., "Association of Type-O Blood with Neuroendocrine Tumors in Multiple Endocrine Neoplasia Type 1," The Journal of Clinical Endocrinology \& Metabolism, vol. 98, no. 1, pp. E109E114, 2013.

[43] S. Hakomori, "Antigen structure and genetic basis of histoblood groups A, B and O: their changes associated with human 
cancer ${ }^{1}$," Biochimica et Biophysica Acta (BBA) - General Subjects, vol. 1473, no. 1, pp. 247-266, 1999.

[44] J. Pendu, S. Marionneau, A. Cailleau-Thomas, J. Rocher, B. Moullac-Vaidye, and M. ClÉMent, "ABH and Lewis histoblood group antigens in cancer," Acta Pathologica, Microbiologica et Immunologica Scandinavica, vol. 109, no. 1, pp. 926, 2001.

[45] D. S. Dede, S. Aksoy, O. Dizdar et al., "Blood ABO groups and risk of breast cancer," Medical Oncology, vol. 27, no. 4, p. 1433, 2010.

[46] S. Y. Miao, W. Zhou, L. Chen, S. Wang, and X. A. Liu, "Influence of ABO blood group and Rhesus factor on breast cancer risk: a meta-analysis of 9665 breast cancer patients and 244768 controls," Asia-Pac J Clin Oncol., vol. 10, no. 2, pp. 101-108, 2014.

[47] J. B. Atkinson, P. C. Tanley, and C. H. Wallas, "Loss of blood group A in acute leukemia: morphologic and biochemical studies of red cells," Transfusion, vol. 27, no. 1, pp. 45-48, 1987.

[48] M. Gharouni, M. Abouzari, A. Rashidi, N. Sodagari, and M. Behzadi, "Low frequency of blood group A in primary central nervous system lymphoma," J Neuro - Oncol., vol. 87, no. 3, pp. 363-364, 2008.

[49] M. Abouzari, M. Behzadi, and A. Rashidi, "Low frequency of blood group A in secondary central nervous system lymphoma," Surgical Neurology International, vol. 3, no. 1, p. 95, 2012.

[50] G. M. Liumbruno and M. Franchini, "Hemostasis, cancer, and ABO blood group: the most recent evidence of association," Journal of Thrombosis and Thrombolysis, vol. 38, no. 2, pp. 160-166, 2014.

[51] Z. Wang, L. Liu, J. Ji et al., "ABO Blood Group System and Gastric Cancer: A Case-Control Study and Meta-Analysis," International Journal of Molecular Sciences, vol. 13, no. 12, pp. 13308-13321, 2012.

[52] C. Rizzato, I. Kato, M. Plummer et al., "Risk of advanced gastric precancerous lesions in Helicobacter pylori infected subjects is influenced by ABO blood group and cagA status," International Journal of Cancer, vol. 133, no. 2, pp. 315-322, 2013.

[53] L. Amundadottir, P. Kraft, R. Z. Stolzenberg-Solomon et al., "Genome-wide association study identifies variants in the ABO locus associated with susceptibility to pancreatic cancer," Nature Genetics, vol. 41, no. 9, pp. 986-990, 2009.

[54] M. Yuan, S. H. Itzkowitz, A. Palekar et al., "Distribution of blood group antigens A, B, H, Lewisa, and Lewisb in human normal, fetal, and malignant colonic tissue," Cancer Research, vol. 45, no. 9, pp. 4499-4511, 1985.

[55] N. Fujitani, Y. Liu, S. Toda, K. Shirouzu, T. Okamura, and $\mathrm{H}$. Kimura, "Expression of $\mathrm{H}$ type 1 antigen of $\mathrm{ABO}$ histoblood group in normal colon and aberrant expressions of $\mathrm{H}$ type 2 and $\mathrm{H}$ type 3/4 antigens in colon cancer," Glycoconjugate Journal, vol. 17, no. 5, pp. 331-338, 2000.

[56] M. I. K. El-Sayed and H. K. Amin, “ABO blood groups in correlation with hyperlipidemia, diabetes mellitus type II, and essential hypertension," Asian Journal of Pharmaceutical and Clinical Research, vol. 8, pp. 236-243, 2015.

[57] P. Greenwell, "Blood group antigens: molecules seeking a function?," Glycoconjugate Journal, vol. 14, no. 2, pp. 159173, 1997.
[58] P. Meyer, R. P. Garay, C. Nazaret et al., "Inheritance of abnormal erythrocyte cation transport in essential hypertension," British Medical Journal, vol. 282, no. 6270, pp. 1114-1117, 1981.

[59] K. G. Tournoy, J. R. Delanghe, D. A. Duprez et al., "Genetic polymorphisms and erythrocyte sodium-lithium countertransport in essential hypertension," Clinica Chimica Acta, vol. 255, no. 1, pp. 39-55, 1996.

[60] T. Nakano, T. Shimanuki, M. Matsushita et al., "Involvement of intestinal alkaline phosphatase in serum apolipoprotein B48 level and its association with $\mathrm{ABO}$ and secretor blood group types," Biochemical and Biophysical Research Communications, vol. 341, no. 1, pp. 33-38, 2006.

[61] G. Fagherazzi, G. Gusto, F. Clavel-Chapelon, B. Balkau, and F. Bonnet, "ABO and Rhesus blood groups and risk of type 2 diabetes: evidence from the large E3N cohort study," Diabetologia, vol. 58, no. 3, pp. 519-522, 2015.

[62] M. Kamil, H. A. N. Al-Jamal, and N. M. Yusoff, "Association of ABO blood groups with diabetes mellitus," Libyan Journal of Medicine, vol. 5, pp. 4847-4850, 2010.

[63] B. H. Athreya and L. L. Coriell, "Relation of blood groups to infection: a survey and review of data suggesting possible relationship between malaria and blood groups," American Journal of Epidemiology, vol. 86, no. 2, pp. 292-304, 1967.

[64] M.-. P. Loscertales, S. Owens, J. O'Donnell, J. Bunn, X. BoschCapblanch, and B. J. Brabin, “ABO Blood Group Phenotypes and Plasmodium falciparum Malaria: Unlocking a Pivotal Mechanism," Advances in Parasitology, vol. 65, pp. 1-50, 2007.

[65] O. A. Iyiola, O. O. Igunnugbemi, U. A. Raheem, and A. T. Anifowoshe, "Gene frequencies of $\mathrm{ABO}$ and $\mathrm{Rh}$ (D) blood group alleles in Ilorin," North-Central Nigeria World Journal of Biological Research, vol. 4, p. 1, 2011.

[66] J. A. Rowe, J. M. Moulds, C. I. Newbold, and L. H. Miller, "P. falciparum rosetting mediated by a parasite-variant erythrocyte membrane protein and complement-receptor 1," Nature, vol. 388, no. 6639, pp. 292-295, 1997.

[67] J. P. Cartron, O. Prou, M. Luilier, and J. P. Soulier, "Susceptibility to invasion by Plasmodium falciparum of some human erythrocytes carrying rare blood group antigens," British Journal of Haematology, vol. 55, no. 4, pp. 639-647, 1983.

[68] M. J. Martin, J. C. Rayner, P. Gagneux, J. W. Barnwell, and A. Varki, "Evolution of human-chimpanzee differences in malaria susceptibility: Relationship to human genetic loss of N-glycolylneuraminic acid," Proceedings of the National Academy of Sciences, vol. 102, no. 36, pp. 12819-12824, 2005.

[69] G. Min-Oo and P. Gros, "Erythrocyte variants and the nature of their malaria protective effect," Cellular Microbiology, vol. 7, no. 6, pp. 753-763, 2005.

[70] J. M. Moulds and J. J. Moulds, "Blood group associations with parasites, bacteria, and viruses," Transfusion Medicine Reviews, vol. 14, no. 4, pp. 302-311, 2000. 